\title{
Estimation of symmetry-constrained Gaussian graphical models: application to clustered dense networks
}

\author{
XIN GAO and HÉLÈNE MASSAM \\ Department of Mathematics and Statistics, York University*
}

June 9, 2014

\section{Some Proofs}

Proof of Theorem 1: This proof is similar to the proof of Theorem 1 in Fan and Li (2001) but is modified for the composite likelihood setting. Consider a ball $\left\|\theta-\theta_{0}\right\|_{2} \leq M n^{-\frac{1}{2}}$, for some finite constant $M$. Using Taylor's expansion and, for $j \in(\mathcal{E} \cup \mathcal{V})$, looking at $p_{\lambda_{n}}(\theta)$ as a function $p_{\lambda_{n}}\left(\left|\theta_{j}\right|\right)$ of $\theta_{j}$, we obtain:

$$
\begin{aligned}
-\partial Q(\theta) / \partial \theta_{j} & =\partial \ell_{c}(\theta) / \partial \theta_{j}-n p_{\lambda_{n}}^{\prime}\left(\left|\theta_{j}\right|\right) \operatorname{sign}\left(\theta_{j}\right) \\
& =\partial \ell_{c}\left(\theta_{0}\right) / \partial \theta_{j}+\sum_{j^{\prime} \in(\mathcal{E} \cup \mathcal{V})}\left(\theta_{j^{\prime}}-\theta_{j^{\prime} 0}\right) \partial^{2} \ell_{c}\left(\theta^{*}\right) / \partial \theta_{j} \partial \theta_{j^{\prime}}-n p_{\lambda_{n}}^{\prime}\left(\left|\theta_{j}\right|\right) \operatorname{sign}\left(\theta_{j}\right),
\end{aligned}
$$

where $\theta^{*}$ is between $\theta$ and $\theta_{0}$. As $E\left(\partial \ell_{c}\left(\theta_{0}\right) / n \partial \theta_{j}\right)=0$ and $\operatorname{var}\left(\partial \ell_{c}\left(\theta_{0}\right) / n \partial \theta_{j}\right)<+\infty$, we have that $\partial \ell_{c}\left(\theta_{0}\right) / \partial \theta_{j}=O_{p}\left(n^{\frac{1}{2}}\right)$. As $\left\|\theta^{*}-\theta\right\|_{2} \leq M n^{-\frac{1}{2}}$ and according to Lemma 1 below, we have $\partial^{2} \ell_{c}\left(\theta^{*}\right) / \partial \theta_{j} \partial \theta_{j^{\prime}}=O_{p}(n)$ component-wise. Because $\theta_{j^{\prime}}-\theta_{j^{\prime} 0}$ are all of $O_{p}\left(n^{-\frac{1}{2}}\right)$, the first two terms of the right-hand side of (1) are of order $O_{p}\left(n^{1 / 2}\right)$.

Next we consider the third term of the right-hand side of (1). When $j \in z^{c}$, for a given $\lambda_{n}$, $\lim _{|\theta|_{j} \rightarrow 0^{+}} p_{\lambda_{n}}^{\prime}\left(\left|\theta_{j}\right|\right) / \lambda_{n}=1$ and this third term can be rewritten as

$$
-n \lambda_{n} \operatorname{sign}\left(\theta_{j}\right) p_{\lambda_{n}}^{\prime}\left(\left|\theta_{j}\right|\right) / \lambda_{n}=-n \lambda_{n} \operatorname{sign}\left(\theta_{j}\right) .
$$

As, for SCAD, lim inf $\operatorname{Si\infty }_{n \rightarrow \infty} \lim \inf _{\left|\theta_{j}\right| \rightarrow 0+} p_{\lambda_{n}}^{\prime}\left(\left|\theta_{j}\right|\right) / \lambda_{n}>0$, and $\sqrt{n} \lambda_{n} \rightarrow \infty$, the third term's asymptotic order is higher than $O_{p}\left(n^{\frac{1}{2}}\right)$ and dominates the first two terms. Thus the sign of $\partial Q(\theta) / \partial \theta_{j}$ is completely determined by the $\operatorname{sign}$ of $\theta_{j}$. This entails that inside this $M n^{-1 / 2}$ neighborhood of $\theta_{0}$, $\partial Q(\theta) / \partial \theta_{j}>0$, when $\theta_{j}>0$ and $\partial Q(\theta) / \partial \theta_{j}<0$, when $\theta_{j}<0$. Therefore for any local minimizer $\hat{\theta}$ inside this ball, we have $\hat{\theta}_{j}=0$ with probability tending to one.

\footnotetext{
${ }^{*}$ The authors gratefully acknowledge the support of their respective NSERC Discovery grants.
} 
Let us now look at what happens when $j \in z$. As $p_{\lambda_{n}}(0)=0$, we obtain

$$
\begin{aligned}
Q\left(\theta_{0}\right)-Q(\theta) & =\ell_{c}(\theta)-\ell_{c}\left(\theta_{0}\right)-n \sum_{j \in(\mathcal{E} \cup \mathcal{V})}\left(p_{\lambda_{n}}\left(\left|\theta_{j}\right|\right)-p_{\lambda_{n}}\left(\left|\theta_{j 0}\right|\right)\right) \\
& \leq\left(\theta-\theta_{0}\right)^{T} \frac{\partial \ell_{c}\left(\theta_{0}\right)}{\partial \theta}+\left(\theta-\theta_{0}\right)^{T} \frac{\partial^{2} \ell_{c}\left(\theta^{*}\right)}{\partial \theta^{2}}\left(\theta-\theta_{0}\right) \\
& -n \sum_{j \in z}\left(p_{\lambda_{n}}^{\prime}\left(\left|\theta_{j 0}\right|\right) \operatorname{sign}\left(\theta_{j 0}\right)\left(\theta_{j}-\theta_{j 0}\right)+p_{\lambda_{n}}^{\prime \prime}\left(\left|\theta_{j 0}\right|\right)\left(\theta_{j}-\theta_{j 0}\right)^{2}(1+o(1))\right) .
\end{aligned}
$$

For $n$ large enough and $\theta_{j 0} \neq 0, \lim _{n \rightarrow+\infty} p_{\lambda_{n}}^{\prime}\left(\left|\theta_{j 0}\right|\right)=0$ and $\lim _{n \rightarrow+\infty} p_{\lambda_{n}}^{\prime \prime}\left(\left|\theta_{j 0}\right|\right)=0$, and the third term of (2) is therefore negligible. From the proof of Lemma 1, it is seen that $\partial^{2} \ell_{c}(\theta) / \partial \theta^{2}$ is continuous in $\theta$. Because $\left\|\theta^{*}-\theta\right\|_{2} \leq M n^{-\frac{1}{2}}$, by the continuity of $\partial^{2} \ell_{c}\left(\theta^{*}\right) / \partial \theta^{2}$, we have that $\frac{1}{n} \partial^{2} \ell_{c}\left(\theta^{*}\right) / \partial \theta^{2}$ converges in probability to $-H\left(\theta_{0}\right)$, which is negative definite. Comparing the first two terms, we can choose $M$ large enough so that the second negative term is dominating the first one. Thus, we have $Q\left(\theta_{0}\right) \leq Q(\theta)$ with probability tending to one for $\theta$ on the unit ball. This implies there exists a local minimizer $\hat{\theta}$ such that $\left\|\hat{\theta}-\theta_{0}\right\|_{2}=O_{p}\left(n^{-1 / 2}\right)$.

Proof to Theorem 2: Based on Taylor expansion presented in Proof to Theorem 1, we have

$$
0=\frac{-\partial Q(\hat{\theta})}{\partial \theta_{z}}=\frac{\partial \ell_{c}\left(\theta_{0}\right)}{\partial \theta_{z}}+\frac{\partial^{2} \ell_{c}\left(\theta^{*}\right)}{\partial \theta_{z} \partial \theta_{z}^{T}}\left(\hat{\theta}_{z}-\theta_{z 0}\right)-n b_{1}-n\left(\Sigma_{1}^{*}\right)\left(\hat{\theta}_{z}-\theta_{z 0}\right)
$$

where $\Sigma_{1}^{*}=\operatorname{diag}\left(p_{\lambda_{n}}^{\prime \prime}\left(\left|\theta_{j}^{*}\right|\right), j \in z\right.$ with $\theta_{j}^{*}$ between $\hat{\theta}_{j}$ and $\theta_{j 0}$. As $\hat{\theta} \rightarrow \theta_{0}$ in probability, $\frac{1}{n} \frac{-\partial^{2} \ell_{c}\left(\theta^{*}\right)}{\partial \theta_{z} \partial \theta_{z}^{T}} \rightarrow$ $H_{z z}$ in probability. The limiting distribution of $\frac{1}{\sqrt{n}} \frac{\partial \ell_{c}\left(\theta_{0}\right)}{\partial \theta_{z}}$ is $N\left\{0, V_{z z}\right\}$. According to Slutsky's theorem, we have $\sqrt{n}\left(H_{z z}+\Sigma_{1}^{*}\right)\left\{\hat{\theta}_{z}-\theta_{z 0}+\left(H_{z z}+\Sigma_{1}^{*}\right)^{-1} b_{1}\right\} \rightarrow N\left\{0, V_{z z}\right\}$. As both $\Sigma_{1}^{*}$ and $b_{1}$ will asymptotically diminish to zero, we have $\sqrt{n} H_{z z}\left(\hat{\theta}_{z}-\theta_{z 0}\right) \rightarrow N\left\{0, V_{z z}\right\}$, as $n \rightarrow \infty$.

\section{Derivations for the updating formula under the RCON model}

Differentiating the composite loglikelihood, we obtain the first derivative of the objective function with respect to the edge class parameter $\theta_{E_{s}}$ as follows:

$$
\begin{aligned}
\frac{\partial Q(\theta)}{\partial \theta_{E_{s}}}= & \left(\sum_{j=1}^{p} \sum_{i ;(i, j) \in E_{s}} \sum_{l ;(l, j) \in E_{s}} \theta_{j j}^{-1} X_{(i)}^{T} X_{(l)}\right) \theta_{E_{s}}+ \\
& \sum_{j=1}^{p}\left(\sum_{i ;(i, j) \in E_{s}} X_{(j)}^{T} X_{(i)}+\theta_{j j}^{-1} \sum_{i ;(i, j) \in E_{s}} \sum_{l ;(l, j) \in E_{s}^{c}} X_{(i)}^{T} X_{(l)} \theta_{l j}\right)+n \lambda \operatorname{sign}\left(\theta_{E_{s}}\right),
\end{aligned}
$$

where $E_{s}^{c}=\left\{(i, j) \mid i \neq j\right.$ and $\left.(i, j) \notin E_{s}\right\}$. 
Now, let us differentiate $Q(\theta)$ with respect to $\theta_{V_{m}}$. Using the fact that $-X B_{j}=\sum_{k \neq j} \theta_{k j} \theta_{j j}^{-1} X_{(k)}$, the likelihood equation for $\theta_{V_{m}}$ is

$$
\begin{aligned}
\frac{\partial Q(\theta)}{\partial \theta_{V_{m}}} & =\frac{1}{2} \sum_{j \in V_{m}}\left[-n \theta_{j j}^{-1}+\left(X_{(j)}-X B_{j}\right)^{t}\left(X_{(j)}-X B_{j}\right)-2 \theta_{j j}^{-1}\left(X_{(j)}-X B_{j}\right)^{t} \sum_{k \neq j} \theta_{k j} X_{(k)}\right] \\
& =\frac{1}{2} \sum_{j \in V_{m}}\left[-n \theta_{j j}^{-1}+\left(X_{(j)}-X B_{j}\right)^{t}\left(X_{(j)}-X B_{j}\right)+2\left(X_{(j)}-X B_{j}\right)^{t} X B_{j}\right] \\
& =\frac{1}{2} \sum_{j \in V_{m}}\left[-n \theta_{j j}^{-1}+X_{(j)}^{t} X_{(j)}-B_{j}^{t} X^{t} X B_{j}\right]=\frac{1}{2} \sum_{j \in V_{m}}\left[-n \theta_{j j}^{-1}+X_{(j)}^{t} X_{(j)}\right. \\
& =\frac{n}{2}\left[\left(\sum_{j \in V_{m}} C_{j j} \sum_{k \neq j} \sum_{l \neq j} \theta_{k j} \theta_{l j} X_{(k)}^{t} X_{(l)}\right]\right.
\end{aligned}
$$

where $q_{j}=\sum_{k \neq j} \sum_{l \neq j} \theta_{k j} \theta_{l j} C_{k l}=\theta_{j j}^{2} B_{j}^{t} X^{t} X B_{j} / n$ and $\left|V_{m}\right|$ is the cardinality of $V_{m}$.

\section{Derivations for the updating formula under the RCOR model}

From the expression of the composite loglikelihood and the fact that $\theta_{j j}^{-1} \theta_{k j}=\left(\frac{\theta_{k k}}{\theta_{j j}}\right)^{1 / 2} \frac{\theta_{k j}}{\sqrt{\theta_{j j} \theta_{k k}}}=$ $-\left(\frac{\theta_{k k}}{\theta_{j j}}\right)^{1 / 2} \rho_{k j}$, we see that

$$
\begin{aligned}
-\ell_{c}\left(\Theta_{D}, \rho\right) & =\frac{1}{2} \sum_{j=1}^{p}\left[-n \log \theta_{j j}+\theta_{j j}\left\|X_{j}-\sum_{j \neq k}\left(\frac{\theta_{k k}}{\theta_{j j}}\right)^{1 / 2} \rho_{k j} X_{k}\right\|^{2}\right] \\
& =\frac{1}{2} \sum_{j=1}^{p}\left[-n \log \theta_{j j}+\theta_{j j}\left\|X_{(j)}-\sum_{\substack{k \in V_{m} \\
k \neq j}} \rho_{k j} X_{(k)}-\theta_{j j}^{-1 / 2} \sum_{k \notin V_{m}} \theta_{k k}^{1 / 2} \rho_{k j} X_{(k)}\right\|^{2}\right]
\end{aligned}
$$

Differentiating the objective function with respect to $\rho_{E_{s}}$, we obtain

$$
\begin{aligned}
\frac{\partial Q\left(\theta_{D}, \rho\right)}{\partial \rho_{E_{s}}}= & \frac{1}{2} \sum_{j=1}^{p}\left[2 \theta_{j j}\left(X_{(j)}-\sum_{k \neq j}\left(\frac{\theta_{k k}}{\theta_{j j}}\right)^{1 / 2} \rho_{k j} X_{(k)}\right)^{t}\left(-\sum_{(j, l) \in E_{s}}\left(\frac{\theta_{l l}}{\theta_{j j}}\right)^{1 / 2} X_{(l)}\right)\right]+n \lambda \operatorname{sign}\left(\rho_{E_{s}}\right) \\
= & \sum_{j=1}^{p}\left[-\sum_{(j, k) \in E_{s}}\left(\theta_{k k} \theta_{j j}\right)^{1 / 2} X_{(j)}^{t} X_{(k)}+\rho_{E_{s}}\left(\sum_{(j, k) \in E_{s}} \theta_{k k}^{1 / 2} X_{(k)}\right)^{t}\left(\sum_{(j, l) \in E_{s}} \theta_{l l}^{1 / 2} X_{(l)}\right)\right. \\
& \left.+\left(\sum_{(j, k) \in E_{s}^{c}} \theta_{k k}^{1 / 2} \rho_{k j} X_{(k)}\right)^{t}\left(\sum_{(j, l) \in E_{s}} \theta_{l l}^{1 / 2} X_{(l)}\right)\right]+n \lambda \operatorname{sign}\left(\rho_{E_{s}}\right) .
\end{aligned}
$$


Differentiating the objective function with respect to $\theta_{V_{m}}$, we obtain

$$
\begin{aligned}
& \frac{\partial-\ell\left(\theta_{D}, \rho\right)}{\partial \theta_{V_{m}}} \\
= & -\frac{n}{2}\left|V_{m}\right| \theta_{V_{m}}^{-1}+\frac{1}{2} \sum_{j \in V_{m}}\left[\left(X_{(j)}-\sum_{k \in V_{m}} \rho_{k j} X_{(k)}\right)^{t}\left(X_{(j)}-\sum_{k \in V_{m}} \rho_{k j} X_{(k)}\right)\right. \\
& \left.-\theta_{j j}^{-1 / 2}\left(\sum_{k \in V_{m}^{c}} \theta_{k k}^{1 / 2} \rho_{k j} X_{(k)}\right)^{t}\left(X_{(j)}-\sum_{k \in V_{m}} \rho_{k j} X_{(k)}\right)\right] \\
= & \frac{n}{2}\left\{-\left|V_{m}\right| y^{2}+b y+a\right\},
\end{aligned}
$$

where $y=\theta_{V_{m}}^{-1 / 2}$,

$$
\begin{aligned}
b & =\frac{1}{n}\left(-\sum_{j \in V_{m}} \sum_{k \in V_{m}^{c}} \theta_{k k}^{1 / 2} \rho_{k j} X_{(k)}^{t} X_{(j)}+\sum_{j \in V_{m}} \sum_{k \in V_{m}^{c}} \sum_{l \neq j, l \in V_{m}} \theta_{k k}^{1 / 2} \rho_{k j} \rho_{l j} X_{(k)}^{t} X_{(l)}\right) \\
& =-2 \operatorname{tr}\left(T^{V_{m}} C \Sigma^{-1 / 2} T^{V_{m}^{c}} \tilde{P}\right)+\operatorname{tr}\left(\tilde{P} T^{v_{m}^{c}} \Sigma^{-1 / 2} C T^{V_{m}} \tilde{P}\right),
\end{aligned}
$$

and

$$
\begin{aligned}
a & =\frac{1}{n}\left(\sum_{j \in V_{m}} X_{(j)}^{t} X_{(j)}-2 \sum_{j \in V_{m}} \sum_{k \in V_{m}, k \neq j} \rho_{k j} X_{(j)}^{t} X_{(k)}+\sum_{j \in V_{m}} \sum_{k \neq j, k \in V_{m}} \sum_{l \neq j, l \in V_{m}} \rho_{k j} \rho_{l j} X_{(k)}^{t} X_{(l)}\right) \\
& \left.=\operatorname{tr}\left(T^{V_{m}} C\right)-2 \operatorname{tr}\left(T^{V_{m}} C T^{V_{m}} \tilde{P}\right)\right)+\operatorname{tr}\left(\tilde{P} T^{V_{m}} C T^{V_{m}} \tilde{P}\right) .
\end{aligned}
$$

Lemma 1. All the second partial derivatives of the log composite likelihood $\partial^{2} \ell_{c}(\theta) / \partial \theta^{2}$ are $O_{p}(n)$.

Proof of Lemma 1: From the expression of $\frac{\partial Q(\theta)}{\partial \theta_{E_{s}}}$ in Section 3.1, we have that

$$
\begin{aligned}
-\frac{\partial \ell_{c}(\theta)}{\partial \theta_{E_{s}}}= & \left(\sum_{j=1}^{p} \sum_{i ;(i, j) \in E_{s}} \sum_{l ;(l, j) \in E_{s}} \theta_{j j}^{-1} X_{(i)}^{T} X_{(l)}\right) \theta_{E_{s}}+ \\
& \left(\sum_{j=1}^{p} X_{(j)}^{T}\left(\sum_{i ;(i, j) \in E_{s}} X_{(i)}\right)+\theta_{j j}^{-1} \sum_{i ;(i, j) \in E_{s}} \sum_{l ;(l, j) \in E_{s}^{c}} X_{(i)}^{T} X_{(l)} \theta_{l j}\right)
\end{aligned}
$$

Differentiating (4), with respect to $\theta_{s}$ again, we obtain

$$
-\frac{\partial^{2} \ell_{c}(\theta)}{\partial \theta_{E_{s}}^{2}}=\left(\sum_{j=1}^{p} \sum_{i ;(i, j) \in E_{s}} \sum_{l ;(l, j) \in E_{s}} \theta_{j j}^{-1} X_{(i)}^{T} X_{(l)}\right)
$$

Differentiating (4), with respect to $\theta_{s^{\prime}}$, we obtain

$$
-\frac{\partial^{2} \ell_{c}(\theta)}{\partial \theta_{E_{s}} \partial \theta_{E_{s^{\prime}}}}=\left(\sum_{j=1}^{p} \sum_{i ;(i, j) \in E_{s}} \sum_{l ;(l, j) \in E_{s^{\prime}}} \theta_{j j}^{-1} X_{(i)}^{T} X_{(l)}\right)
$$


Differentiating (4), with respect to $\theta_{V_{m}}$, we obtain

$$
\begin{aligned}
-\frac{\partial^{2} \ell_{c}(\theta)}{\partial \theta_{E_{s}} \partial \theta_{V_{m}}}= & \left(-\sum_{j \in V_{m}} \sum_{i ;(i, j) \in E_{s}} \sum_{l ;(l, j) \in E_{s}} \theta_{j j}^{-2} X_{(i)}^{T} X_{(l)}\right) \theta_{E_{s}}- \\
& \left(\sum_{j \in V_{m}} \theta_{j j}^{-2} \sum_{i ;(i, j) \in E_{s}} \sum_{l ;(l, j) \in E_{s}^{c}} X_{(i)}^{T} X_{(l)} \theta_{l j}\right)
\end{aligned}
$$

From the expression of $\frac{\partial Q(\theta)}{\partial \theta_{V_{m}}}$ in Section 3.1, we have that

$$
-\frac{\partial \ell_{c}(\theta)}{\partial \theta_{V_{m}}}=\frac{1}{2} \sum_{j \in V_{m}}\left[-n \theta_{j j}^{-1}+X_{(j)}^{t} X_{(j)}-\theta_{j j}^{-2} \sum_{k \neq j} \sum_{l \neq j} \theta_{k j} \theta_{l j} X_{(k)}^{t} X_{(l)}\right]
$$

Differentiating (5), with respect to $\theta_{V_{m}}$ again, we obtain

$$
-\frac{\partial^{2} \ell_{c}(\theta)}{\partial \theta_{V_{m}}^{2}}=\frac{1}{2} \sum_{j \in V_{m}}\left[n \theta_{j j}^{-2}+2 \theta_{j j}^{-3} \sum_{k \neq j} \sum_{l \neq j} \theta_{k j} \theta_{l j} X_{(k)}^{t} X_{(l)}\right]
$$

Differentiating (5), with respect to $\theta_{V_{m^{\prime}}}$, we clearly obtain 0 . Because the expectation of any sum of the type $\sum_{i} \sum_{k} X_{(i)}^{t} X_{(k)}$ is $n$ times the corresponding block of $E(C)=E\left(X^{t} X\right)$ where $X$ is the data matrix defined in Section 3, and since the only term in the derivatives above without a sum of the type $\sum_{i} \sum_{k} X_{(i)}^{t} X_{(k)}$ is $\frac{1}{2} \sum_{j \in V_{m}}\left[-n \theta_{j j}^{-1}\right]$, we have each element of $\partial^{2} \ell_{c}(\theta) / \theta^{2}$ is $O_{p}(n)$.

\section{The analysis of the "Math" data set}

We analyze the "math" data set from Mardia, Kent, and Bibby (1979), which gives the grades of 88 students in 5 different mathematics subjects: Mechanics (me), Vectors (ve), Algebra (al), Analysis (an) and Statistics (st). The model with symmetries proposed by Højsgaard and Lauritzen (2008) has vertex colour classes $\{\mathrm{al}\},\{\mathrm{me}, \mathrm{st}\},\{\mathrm{ve}, \mathrm{an}\}$ and edge colour classes $\{(\mathrm{al}, \mathrm{an})\}$, $\{(\mathrm{an}, \mathrm{st})\},\{(\mathrm{me}, \mathrm{ve}),(\mathrm{me}, \mathrm{al})\}$, and $\{(\mathrm{ve}, \mathrm{al}),(\mathrm{al}, \mathrm{st})\}$. Since the dimension of the data is low, we perform composite likelihood estimation on this symmetric model with no penalty imposed on the parameters. The tolerance level of the iterations in the composite likelihood estimation algorithm is set to be $10^{-6}$. In Table 1, the composite likelihood estimates are compared with the maximum likelihood estimator and a naive estimator. The naive estimator estimates the edge class parameters and vertex class parameters by simply averaging all the values belonging to the same class in the inverse sample covariance matrix. The standard errors of the composite likelihood estimates and the naive estimates are obtained from the bootstrap procedure. The bootstrap sample size is 88 and the number of bootstrap samples is 1000. The three methods yield similar but different values. The composite likelihood estimates are closer to the maximum likelihood estimates than to the naive estimates. In the analysis of Table 1 which is based on the graphical structure given in Højsgaard and Lauritzen (2008), we assume that we know the edge classes and also know which classes have zero edges. So in the update of the parameters, the groups of zero edges are set to be zero and not estimated. 
Table 1: Comparison of full likelihood, composite likelihood, and moment estimates on the analysis of "math" dataset

\begin{tabular}{ccccccc}
\hline \hline parameter & est & std & est & std $_{b}$ & est & std $_{b}$ \\
\hline \hline likelihood & \multicolumn{3}{c}{ composite } \\
vcc1 & 0.027878 & 0.003653 & 0.027833 & 0.004254 & 0.022482 & 0.004528 \\
vcc3 & 0.010003 & 0.000944 & 0.005873 & 0.000529 & 0.006708 & 0.000653 \\
ecc1 & 0.005843 & 0.000582 & 0.009982 & 0.000945 & 0.011420 & 0.001385 \\
ecc2 & -0.007984 & 0.001537 & -0.007710 & 0.001468 & -0.003170 & 0.001967 \\
ecc3 & -0.001775 & 0.000740 & -0.001899 & 0.000747 & -0.004066 & 0.001029 \\
ecc4 & -0.002951 & 0.000443 & -0.002848 & 0.000466 & -0.002945 & 0.000836 \\
\hline \hline
\end{tabular}

"std" (Column 3) stands for the standard error evaluated from Hessian matrix; "std $b$ (Column 5 and 7 ) stands for the standard error obtained from boostrap method. The bootstrap sample size is 88 and the number of bootstrap samples is 1000 .

\section{References}

Højsgaard, S and Lauritzen, S. L. (2008), "Graphical Gaussian Models with Edge and Vertex symmetries", Journal of the Royal Statistical Society, Series B, 70, 1005-1027.

Mardia, K. V., Kent, J., and Bibby, J (1979), Multivariate analysis, Academic Press, London. 